Soluciones tecnológicas a problemáticas sociales y ciudadanas. El caso de los Retos Cívicos y Públicos en México

Technological Solutions to Social and Citizen Problems. The
Case of Civic and Public Challenges in Mexico

Martin Adalberto Tena-Espinoza-de-los-Monteros

Coordinación de Bibliotecas - Red de Bibliotecas (REBIUdeG), Universidad de Guadalajara. Guadalajara, Jalisco, México. mtenaespinoza@ udgvirtual.udg. $m x$

Resumen

El objetivo de este trabajo es presentar el proceso de innovación cívica que, basado en soluciones tecnológicas y en iniciativas abiertas, propone la organización de la sociedad civil Codeando México para la atención y respuesta a problemáticas sociales y ciudadanas en México.

Se abordan y describen las iniciativas Retos Cívicos y Retos Públicos como experiencias de innovación en la implementación de estrategias tecnológicas para la solución de problemáticas sociales y ciudadanas.

Se reflexiona sobre la adopción cívica de TICs y su irrupción en los procesos de innovación, así como el impacto que tienen en la conformación de un nuevo ecosistema cívico.

Por último, se hace mención de las estrategias de Hacking cívico y Comunidades cívicas que la organización Codeando México promueve como modelo para la vinculación y la participación ciudadana en el marco de la innovación cívica.

Palabras Clave

Participación del ciudadano; Tecnologías de la información Nuevas tecnologías; Desarrollo social; Problemas sociales

\begin{abstract}
This paper describes the process of civic innovation that, based on technological solutions and open initiatives, the civic society's organization Codeando México suggests for the attention and solution of social and civic problems in Mexico.

The Retos Cívicos (Civic Challenges) and Retos Públicos (Public Challenges) initiatives are addressed and described as experiences of innovation in the implementation of technological strategies for the solution of social and civic problems.

A reflection is made on the civic appropriation of the ICTS and its irruption in the processes of innovation, as well as on the impact that the ICTS have in the conformation of a new civic ecosystem

Last, the strategies of Hacking cívico (Civic Hacking) and Comunidades Cívicas (Civic Communities) that the Codeando México organization promotes as a model for the linkage and civic participation within the frame of civic innovation, are mentioned.
\end{abstract}

Keywords

Citizen participation; Information technology; New technologies; Social development; Social problems 


\section{Innovación cívica}

El concepto "innovación" en términos de la Real Academia Española (2014c) es la acción de "mudar o alterar algo, introduciendo novedades". Arenilla-Sáez y García-Vegas (2013) amplían el concepto al asociarlo "a productos y procesos que introducen mejoras significativas en la forma como se resuelven los problemas o se hacen las cosas". Di Siena (2015) lo vincula con las palabras "cambio", "oportunidad" y "mejora", y considera a estas palabras sinónimos y cercanas al concepto.

Las definiciones anteriores nos señalan que una innovación debe cumplir con dos criterios básicos: 1) ser novedosa, aunque no necesariamente original, y 2) generar una mejora que pueda ser traducida tanto en un resultado satisfactorio que denote eficacia, como en una alternativa viable a las soluciones existentes. A estos dos criterios, cabe agregar dos más sugeridos por Phills et al. (2008): 1) ser sostenible o justa y 2) que sea capaz de generar valor.

Por otro lado, el concepto "cívica" alude al sentido de "pertenencia a la ciudad o a los ciudadanos" y es "relativo al civismo" (Real Academia Española, 2014a), el término "civismo" nos refiere a "el comportamiento respetuoso del ciudadano con las normas de convivencia pública" y al "celo por las instituciones e intereses de la patria" (Real Academia Española, 2014b). Una condensación de los cuatro elementos que conforman el concepto de "cívica" sería: el interés ciudadano por la observancia de las instituciones en un marco de desarrollo social, de reconocimiento de derechos y obligaciones, y para la mejora de la convivencia social en el espacio de una comunidad.

Así pues, al conjuntar estos dos conceptos "innovación" y "cívica", en su acepción más simple tendríamos la siguiente definición: la búsqueda y desarrollo de nuevas soluciones más eficaces, eficientes, sostenibles, o simplemente con un mayor valor que las soluciones existentes a problemas vinculados a la ciudad y a los ciudadanos.

\subsection{Las TICs: mecanismos de la innovación cívica}

El advenimiento de las TICs ha trasformado los actuales y futuros entornos y modelos de desarrollo y convivencia en todas sus esferas. La adopción e integración que estas han tenido en mayor o en menor medida, así como el impacto positivo o negativo que han podido generar, ha sido a partir de su papel como agentes vinculantes, capaces de generar productos y procesos atribuibles a acciones y resultados de innovación.

De esta manera, tenemos que la tecnología, al ser adoptada, integrada y utilizada en cada vez más procesos cotidianos del ejercicio y desarrollo ciudadano y de las sociedades, pasa a convertirse en un 
medio, un recurso y una herramienta. Es desde cada uno de estos elementos o desde su conjunción en un todo, que se puede innovar cívicamente.

Así pues, la "innovación cívica" pude definirse como: la búsqueda y desarrollo de nuevas soluciones más eficaces, más eficientes y más sostenibles, que, soportadas en una base tecnológica, estén orientadas a la resolución de problemas sociales y ciudadanos, suponiendo una mejora a las condiciones y procesos sociales y ciudadanos existentes.

\section{Codeando México}

Codeando México es la organización de la sociedad civil mexicana que ha implementado, desarrollado, y hecho escalable el mecanismo y la estrategia de los "retos" (Morato, 2014). La labor y trascendencia de Codeando México se remonta a finales del año 2012 cuando en sus inicios se definió como "un equipo de programadores y diseñadores de software que tomaron la iniciativa de comenzar a hacer hacking cívico" (Wilhelmy, 2013), es decir, desarrollar tecnología de impacto social (tecnología cívica) "para ayudar a resolver los problemas que más atención necesitaban en el país" (Codeando México, 2015).

\subsection{La propuesta inicial: una Plataforma Cívica}

En diciembre de 2012, Codeando México participo en la 2. ${ }^{a}$ edición del megaconcurso regional bajo la modalidad de hackathon "Desarrollando América Latina 2012 - DAL2012" (Arellano-Valdivia, 2012a; DAL, 2012a; Moreno, 2012). En dicha edición participaron 8 países latinoamericanos: Argentina, Bolivia, Brasil, Chile, Costa Rica, México, Perú y Uruguay. Codeando México resultó ganador del hackathon nacional entre los 7 proyectos mexicanos participantes (OpenData.MX, 2012) y obtuvo una "mención honrosa" con la versión "Codeando América Latina" entre las 22 aplicaciones finalistas de cada país y de las cuales surgieron los proyectos ganadores del hackathon a nivel regional (Arellano-Valdivia, 2012b; DAL, 2012b, 2012c).

La propuesta presentada por Codeando México y su versión Codeando América Latina fue una plataforma de innovación abierta "Plataforma Cívica", la cual consistía en un espacio de hackatón permanente en donde se pueda "convocar y publicar proyectos por parte de fundaciones o gente de la sociedad civil, exponiendo datos abiertos e involucrando interesados del área de tecnología, diseño, o de industrias en general para desarrollar una solución tecnológica" y en el cual la "transparencia y colaboración ocurren todos los días" a partir de la "interacción entre organizaciones de la sociedad civil y la comunidad tecnológica para proponer soluciones a temas cívicos" (Arellano-Valdivia, 2012b; Codeando México, 2014a; Codeando América Latina, 2012; DAL, 2012d). 


\subsection{Los primeros “Retos": la irrupción de la \#App1 15 y el \#RetoSATmovil}

Codeando México irrumpió en el escenario político nacional en marzo de 2013 al haber conocido, a través de una investigación periodística, la licitación aprobada por la Cámara de Diputados correspondiente a la LXII Legislatura del Congreso de la Unión- para adquirir una aplicación móvil en \$115 millones de pesos con el fin de monitorear la actividad legislativa de los diputados. Codeando México cuestionó públicamente el costo de dicha aplicación, pues según la opinión de varios especialistas de la industria de software, no debería de ser mayor a \$500 mil pesos. Esta investigación periodística se masificó y discutió en redes sociales bajo el \#App115 (Nava, 2013; Tecayehuatl, 2013).

La investigación periodística se tradujo en una petición en Change.org dirigida a la Cámara de Diputados, la cual solicitaba detener la adquisición de la aplicación móvil. La petición fue respaldada por 1,941 firmas a los pocos días de ser lanzada, el impacto mediático y el apoyo a la petición, logró que la Cámara de Diputados cancelara el contrato de compra de la aplicación (Aguilar, 2013).

A la par de la investigación periodística y la petición ciudadana de frenar la compra, el cuestionamiento de Codeando México se tradujo en una convocatoria a la comunidad de hackers y desarrolladores de software a construir una aplicación open source que respondiese a todas las especificaciones solicitadas por la Cámara de Diputados para su app. La propuesta fue lanzada bajo el nombre "Derrocando a la Mexican Tech Mafia" (Codeando México, 2013). La convocatoria recibió la atención de más de 150 participantes, los cuales desarrollaron 5 apps de código libre, además de desarrollar 3 aplicaciones web para entregar datos y la construcción de una API con datos de los congresistas y las cuales fueron presentadas ante una comitiva de la Cámara de Diputados. Todo esto ocurrió en un lapso de 10 días (Casanueva, 2013; Paniagua, 2013; Saúl-Rodríguez y Morales, 2013).

De esta manera, Codeando México lanzaba su primer reto a la participación abierta y el desarrollo tecnológico para la solución de una problemática deinterés ciudadanoy de observancia gubernamental. Se iniciaba así un movimiento basado en el hacking cívico y la innovación cívica, conformando y desarrollando una joven e incipiente comunidad cívica en el país, la cual estaba convencida de la búsqueda de soluciones tecnológicas a problemáticas sociales como alternativa de cambio, como vía de participación y como herramienta para el empoderamiento ciudadano.

El impacto que tuvo el reto de la \#App115 llamó la atención de ciertos sectores del Gobierno Federal Mexicano, lo que motivó al desarrollo de un primer reto en conjunto entre Codeando México y una agencia federal, el Servicio de Administración Tributaria (SAT). El reto se denominó \#RetoSATmovil (Codeando México, 2013b; Gobierno de México, 2013a; Saavedra, 2013) (ver Figura 1) y se enmarco en la celebración de la "Semana del Emprendedor" (Entrepreneur, 2013; Gobierno de México, 2013b; 
Torreblanca, 2013). Este reto convocaba a rediseñar la app móvil de dicha dependencia, y otorgaba al equipo ganador un contrato para desarrollar el proyecto e implementar la app. Con este segundo reto se daba un primer paso para institucionalizar una nueva forma de colaboración entre el gobierno y un universo diverso de emprendedores integrado por hackers, programadores, tecnólogos y diseñadores; dando paso a un nuevo mecanismo de innovación publica, la innovación cívica (Casanueva, 2013b).

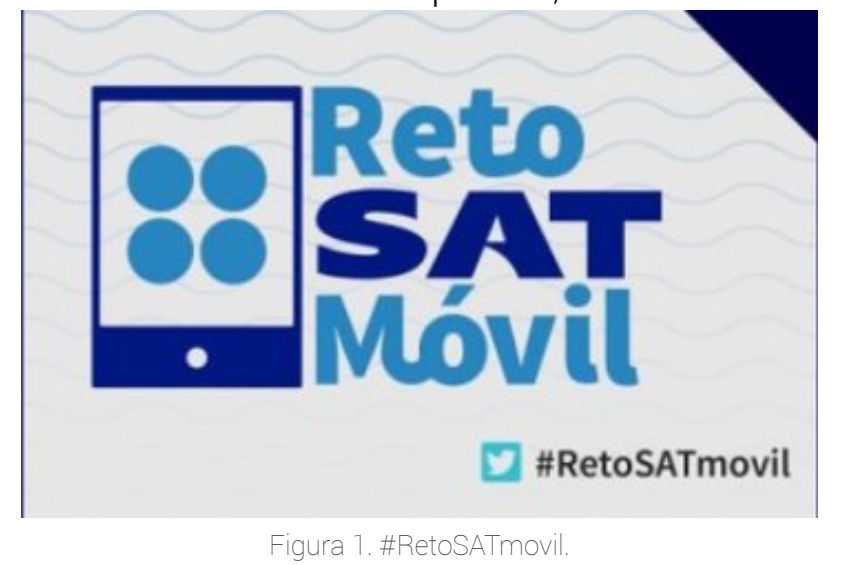

\section{Retos Cívicos}

A raíz de los resultados obtenidos con el lanzamiento de los retos \#App115 y \#RetoSATmovil, y con la firme convicción de mantenerse fieles a la esencia del proyecto originario, el de ser una "Plataforma Cívica", Codeando México puso en marcha una de sus propuestas de innovación cívica, los "Retos Cívicos" (https://codeandomexico.org/). Los "Retos Cívicos" son en esencia un mecanismo para "la innovación abierta y la interacción entre organizaciones de la sociedad civil (OSC) y la comunidad tecnológica del país, y a través del cual se busca crear soluciones a problemáticas cívicas, públicas y sociales" (Codeando México, 2016a).

\subsection{Cómo funcionan los Retos Cívicos}

La plataforma "Retos Cívicos" es lanzada oficialmente en marzo del 2016 (Codeando México, 2016a), ofreciendo a las OSC un espacio en el que presentar a manera de reto, sus problemáticas no resueltas ante equipos y comunidades de tecnología. Estas comunidades trabajan y proponen soluciones tecnológicas a dichas problemáticas (ver Figura 2).

Como resultado de los retos se obtiene para las OSC el desarrollo y la implementación de una solución tecnológica a la problemática presentada, así como un incentivo económico para el equipo de tecnología ganador, el cual en estos momentos es auspiciado por Microsoft. Actualmente los retos a tomar en cuenta para su lanzamiento deben tener incidencia sobre los Objetivos de Desarrollo Sostenible (ODS) y la Agenda 2030 de la ONU (ver Figura 3). 


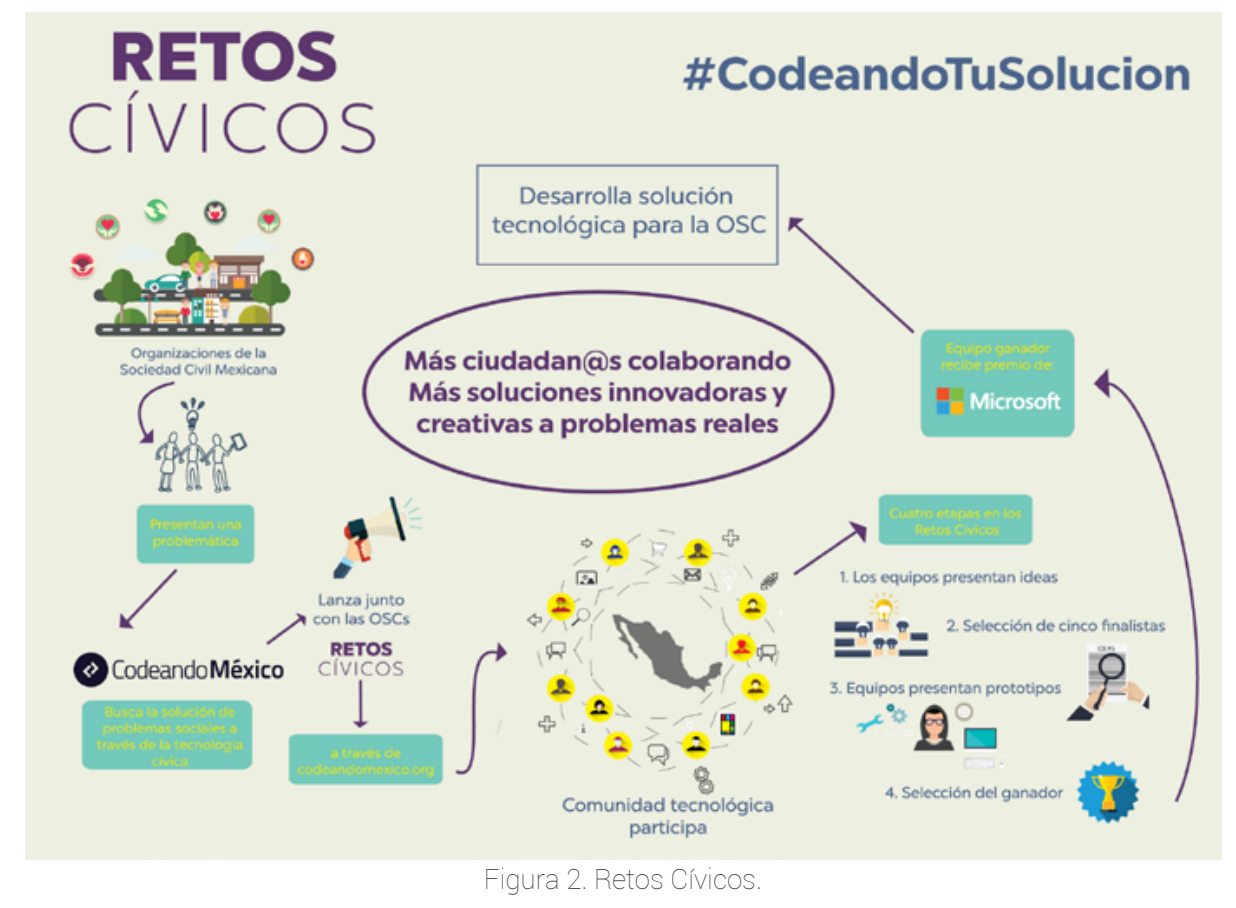

¿Sobre qué temas pueden ser los retos?

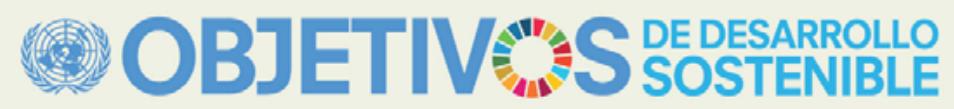
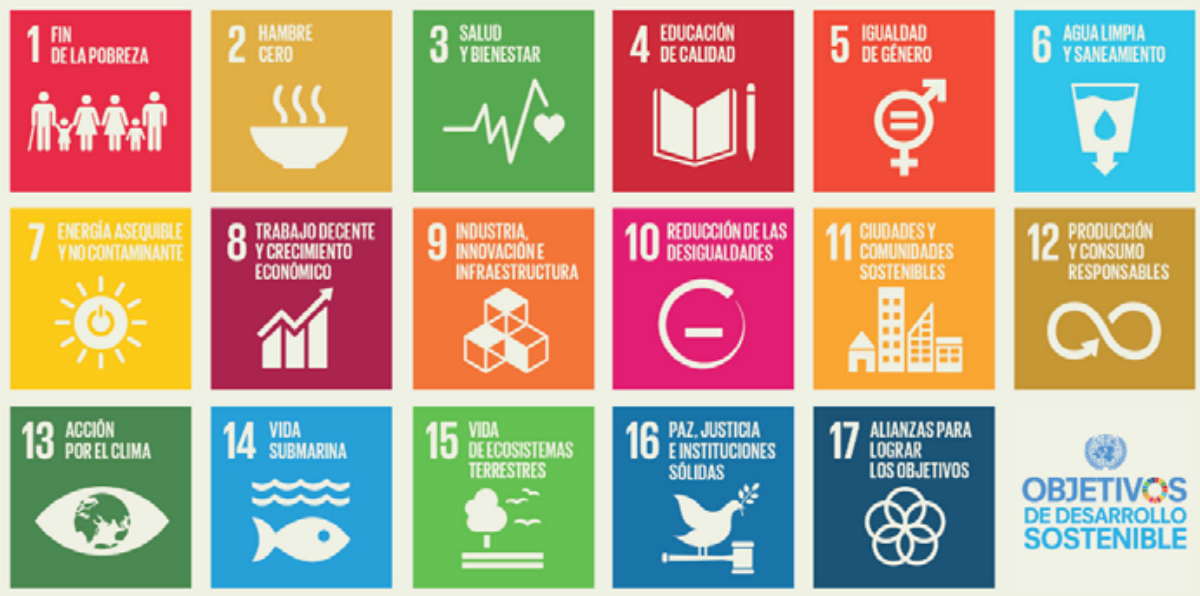

(2)

OBJETIVOS

DEDESARROLLO
SOSTENIBLE

Figura 3. Objetivos de Desarrollo Sostenible.

\subsection{Impactos de los Retos Cívicos}

Desde sus inicios Codeando México y sus distintas comunidades han venido desarrollando y trabajando en diversos Retos Cívicos a través de su plataforma inicial. Prueba de ello son los más de 50 retos de más de 13 organizaciones y los cerca de 1400 participantes que han colaborado en ellos (Salazar, 2016). Sin embargo, es hasta su relanzamiento en marzo de 2016 que las convocatorias se formalizan y se lanzan con un apoyo y patrocinio económico que le permite a las soluciones ganadoras desarrollar las aplicaciones propuestas. Para este relanzamiento, Codeando México diseñó 
una nueva plataforma y estableció nuevos canales de comunicación con sus comunidades y con las organizaciones de la sociedad civil (http://retoscivicos.codeandomexico.org/).

Durante el año 2016, se lanzaron dos convocatorias bajo el nuevo modelo de participación, la primera de ellas en marzo y la segunda en septiembre. En el primero de los retos se trabajó en la solución de la problemática presentada en conjunto por las organizaciones civiles Techo México y Observatorio del Derecho a la Vivienda² (Codeando México, 2016b). Estas organizaciones presentaron como problemática específica el no tener mapeados los asentamientos irregulares de las zonas marginales donde realizan su trabajo como OSC, lo cual les impide identificar y censar riesgos para su posterior atención y solución. El Reto Cívico lanzado para resolver esta problemática fue el desarrollo de un "CoMapp" (Observatorio del Derecho a la Vivienda, 2016). El reto tuvo la participación de 33 propuestas, de estas se seleccionaron cinco finalistas, las cuales pudieron desarrollar sus prototipos. De estos finalistas el ganador fue la startup Eucledian ${ }^{3}$, que actualmente se encuentra desarrollando y testeando la aplicación con los fondos del premio recibido (Codeando México, 2016c).

En el segundo de los retos se trabajó para resolver la carencia de registros digitales de las personas migrantes en tránsito que son acogidas en un centro de atención a migrantes. También se trabajó en solucionar el control y procesamiento digital de las operaciones y servicios que son brindados en el centro de atención. El reto fue presentado por la organización FM4 Paso Libre ${ }^{4}$ (Codeando México, 2016d). Se contó con la participación de 14 propuestas, de las cuales se seleccionaron cinco finalistas para desarrollar los prototipos funcionales. De estos cincos finalistas, la propuesta ganadora fue eCAM (Centro de Atención al Migrante Electrónico) de la consultora de software y laboratorio de innovación wedevelop ${ }^{5}$, la cual se encuentra desarrollando la versión final de la aplicación.

El relanzamiento y la configuración de un modelo integral para el desarrollo y conclusión de los "Retos Cívicos", hace patente una temprana madurez en una organización con apenas cinco años de existencia. También denota la generación de un aprendizaje significativo al lograr conformar un modelo funcional y dinámico capaz de generar y promover la innovación cívica y el desarrollo de tecnología cívica en la solución de problemáticas sociales y ciudadanas en el país. Estas competencias y habilidades como organización y como comunidad le han permitido a Codeando México posicionarse como un referente y un impulsor de iniciativas cívicas, teniendo la capacidad de desarrollar, apoyar y colaborar con otros proyectos de innovación cívica como son: DataMX, Explica la Ley, Reporte Ciudadano, Tu Plataforma Abierta, Desaparecidos, Centro de Apertura de Datos, Educación Ciencia México, Congreso Abierto, entre otros ${ }^{6}, 7$.

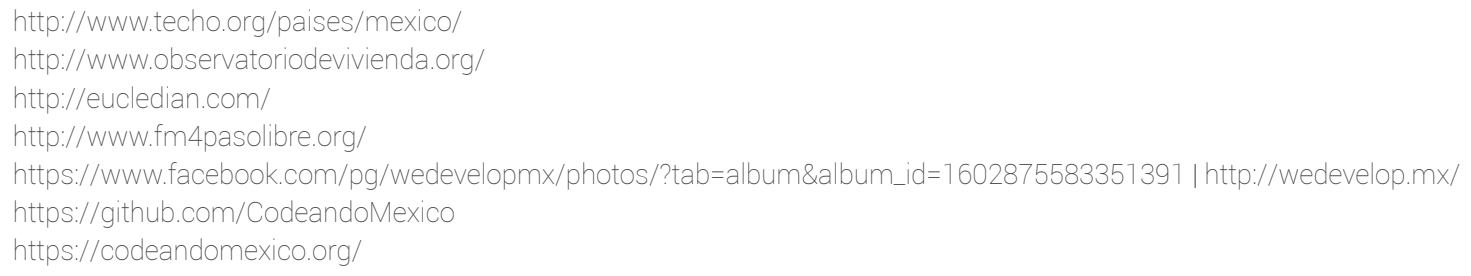




\section{Retos Públicos}

El proyecto de "Retos Públicos" es una iniciativa derivada y configurada a partir de las experiencias que hemos descrito anteriormente, tanto el reto de la \#App115 como el \#RetoSATmovil dieron las actuales pautas para la definición y el desarrollo de un esquema institucional de lo que hoy se conoce como "Retos Públicos".

Con base en dichas experiencias, el Gobierno Federal, a través de la Coordinación de la Estrategia Digital Nacional (CEDN) ${ }^{8}$, con el apoyo del Programa de Política Económica (MEPP) de la Agencia de los Estados Unidos para el Desarrollo Internacional (USAID/México), y en colaboración con Codeando México, diseñó un modelo de contratación del sector público para la adquisición de servicios de tecnología. El modelo se sustenta en el desarrollo de "una plataforma en línea para gestionar convocatorias públicas", donde "pequeñas y medianas empresas puedan participar como proveedoras de tecnología del Gobierno Federal", proponiendo soluciones digitales a necesidades y demandas del sector público (Codeando México, 2014b, 2014c; Gutiérrez y Ocejo, 2015; MEPP, 2014).

Bajo este modelo de contratación, los pequeños y medianos emprendedores tecnológicos tienen la oportunidad de competir dentro de un esquema abierto, dinámico y participativo. Este esquema contribuye a democratizar y transparentar el gasto del Gobierno Federal en la adquisición de servicios y productos de software de pequeña y mediana escala (ver Figura 4).

\section{¿QUE ES RETOS PÚBLICOS?}

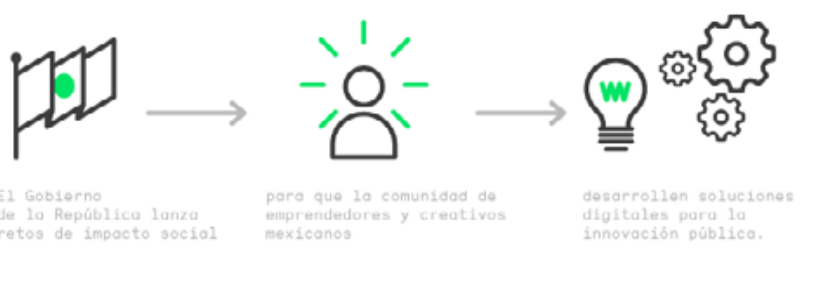

OBJETIVO

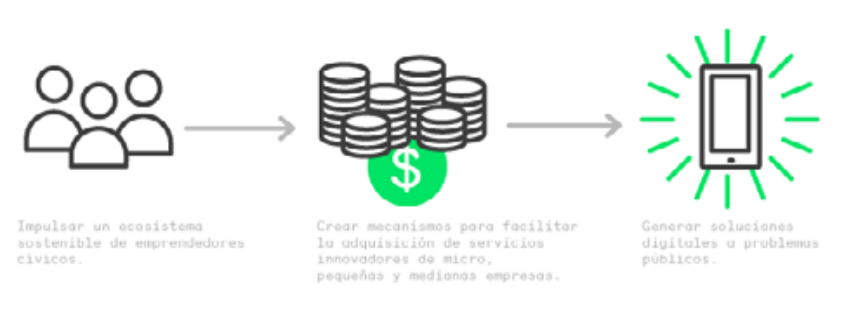

Figura 4. Retos Públicos.

Cabe señalar que para que este modelo de contratación llegara a ser posible, fue necesario realizar adecuaciones a la Ley de Adquisiciones Arrendamientos y Servicios del Sector Público y a su

8 http://www.gob.mx/mexicodigital/ 
reglamento, así como a otras disposiciones referentes a los procesos de contratación establecidos para las instituciones de gobierno (Gobierno de México, 2016a).

\subsection{Sinergias del Proyecto Retos Públicos}

Una de las primerias sinergias que tuvo el proyecto Retos Públicos, fue su vinculación con la Estrategia Digital Nacional (EDN) ${ }^{9}$. En agosto de 2014, en el marco de una nueva edición de la Semana del Emprendedor, el Gobierno de la República, a través de la CEDN, realizó el lanzamiento oficial del proyecto "Retos Públicos" con el "objetivo de estimular la innovación de servicios digitales y fomentar el desarrollo de soluciones tecnológicas innovadoras a los retos que enfrenta el país". Con ello se buscaba contribuir al cumplimiento de los cincos objetivos que sustentan la EDN: 1) Transformación gubernamental, 2) Economía digital, 3) Educación de calidad, 4) Salud universal y efectiva y 5) Seguridad ciudadana (Gobierno de México, 2014a).

La segunda de las sinergias del proyecto Retos Públicos fue su inclusión como parte de las 25 acciones que integran los cinco compromisos del "Plan de Acción 2013-2015 México de la Alianza para el Gobierno Abierto en México (PA15)"10, en el marco de la iniciativa global The Open Government Partnership ${ }^{11}$. En dicho documento, el proyecto Retos Públicos es una de las dos acciones a desarrollar como parte del compromiso N. ${ }^{\circ}$ 4: Empoderamiento y Participación Ciudadana. Con este compromiso, el Gobierno Federal busca "promover la capacidad de los ciudadanos para participar en los asuntos públicos, transformando la relación gobierno-sociedad" (Gobierno de México, 2014b).

Es así como el proyecto Retos Públicos con estas sinergias se convierte en una herramienta y un mecanismo gubernamental que busca "solucionar problemas públicos a partir de que las dependencias de gobierno abran sus procesos a la innovación cívica para generar la apertura de datos y la creación de un ecosistema de emprendedores cívicos" (Gobierno de México, 2014c).

\subsection{Cómo funcionan los Retos Públicos}

Los Retos Públicos son llamados del gobierno federal para presentar propuestas de solución tecnológica a problemas y demandas de impacto social que la administración pública propone. El reto es lanzado a partir de convocatorias abiertas para que empresas y emprendedores que tengan la capacidad y las competencias necesarias para desarrollar software a escala media (facturación menor a $\$ 4,000,000 \mathrm{MXN}$ ), compitan para ganarse un contrato como proveedor, para desarrollar la solución digital que propusieron al reto lanzado por la dependencia o institución del gobierno federal (ver Figura 5).

\footnotetext{
9 http://www.gob.mx/mexicodigital/

$10 \quad$ http://www.pa2015.mx/

11 http://www.opengovpartnership.org/
} 


\section{PROCESO}

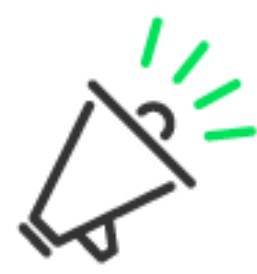

SE LANZA

CONVOCATORIA

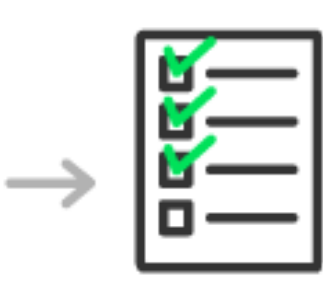

EVALUACIÓN DE PROPUESTAS

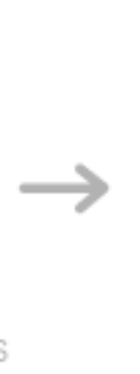

Reciben apoyos para desarrollar

Prototipos

Funcionales

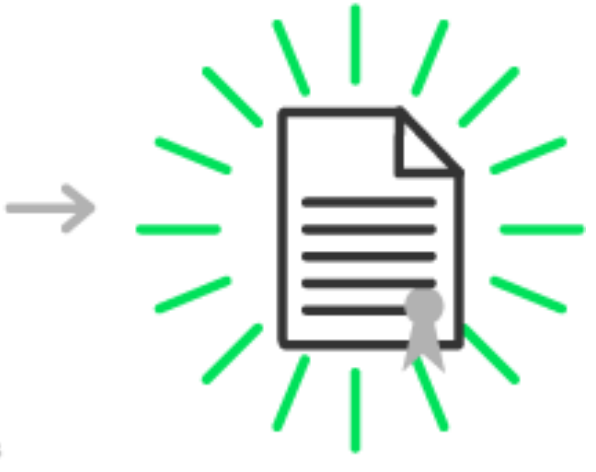

GANADOR DEL CONTRATO
Propuestas

a nivel idea

ETAPA

\section{1}

ETAPA

\section{2}

Figura 5. Proceso de los Retos Públicos.

A través de este esquema de innovación publica, el gobierno federal busca apoyar a pequeños desarrolladores y programadores de software con la intención de detonar el desarrollo de la industria de la innovación a nivel de las pequeñas y medianas empresas (PyMEs) del sector, así como disminuir el costo en las compras TICs que realiza el gobierno.

\subsection{Avances de los Retos Públicos}

A dos años de su lanzamiento oficial, el portal del Proyecto Retos Públicos había convocado al desarrollo de 15 retos provenientes de 12 instancias del Gobierno Federal (ver Figura 6).

Con motivo del 4. ${ }^{\circ}$ Informe de Gobierno Federal, la CEDN reportaba la participación de más de 2,500 emprendedores y pequeñas empresas de base tecnológica, los cuales habían presentado un total de 1,700 propuestas a partir de las cuales se han generado 75 prototipos funcionales (Gobierno de México, 2016b). Además del alcance y el impacto que los Retos Públicos ha tenido en el Gobierno Federal, la iniciativa de Retos Públicos actualmente está siendo replicada y adoptada en diversas instancias de los gobiernos estatales y municipales del país, siendo uno de los casos más recientes el de Retos Jalisco (Gobierno Estatal) y Desafío San Pedro (Gobierno Municipal) (Gobierno de Jalisco, 2016; Gobierno de San Pedro Garza García, 2016). 


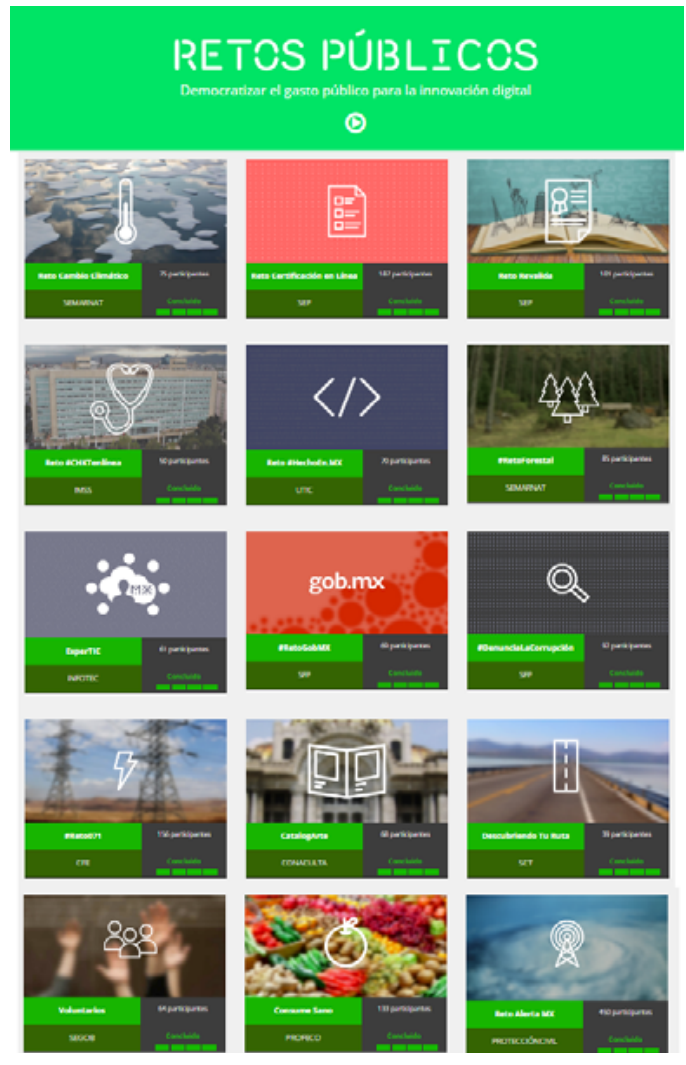

Figura 6. Retos Públicos al 2016.

\section{Un nuevo ecosistema cívico}

A través de los ejemplos y la experiencia descrita, resulta evidente la conformación y desarrollo de un nuevo entorno de confluencia e interacciones para diversos actores: ciudadanos, hackers cívicos, desarrolladores de software, periodistas, investigadores, organizaciones de la sociedad civil, fundaciones, empresas y gobierno. Este nuevo entorno está conformado y determinado por el uso, la apropiación y el desarrollo de recursos y herramientas tecnológicas. Las interrelaciones que suceden en dicho entorno, entre dichos actores y entre el consumo y el uso de recursos y herramientas tecnológicas, dan origen a un nuevo ecosistema, un ecosistema cívico tecnológico.

Este nuevo ecosistema cívico con base tecnológica, viene a afrontar e innovar el gastado y cada vez más infructuoso y vejado ecosistema de participación ciudadana vigente. Si nos permitimos utilizar una analogía para explicar la conformación de este nuevo ecosistema cívico tecnológico, sobre el antiguo ecosistema de participación ciudadana, bien cabría emplear el proceso de sucesión ecológica que se da en los sistemas biológicos, y el cual consiste en: "el proceso de cambio que sufre un ecosistema en el tiempo, como consecuencia de los cambios que se producen tanto en las condiciones del entorno como en las poblaciones que lo integran, dando lugar a un ecosistema cada vez más organizado y complejo" (Odum, 1969). 
Así pues, esta sucesión y proceso de cambio que se ejemplifica en la analogía, puede emplearse en el entendimiento del proceso transición que vive la innovación pública hacia una innovación cívica, la cual busca dar solución a viejos problemas sociales y ciudadanos, generando mejoras de formas novedosas, eficaces, autónomas a partir de una base tecnológica y abierta.

\section{Conclusiones}

Hemos visto cómo dos iniciativas con un origen común; el de resolver una problemática social o ciudadana a partir de su abordaje como un reto, y su confrontación y tratamiento a partir de los intereses y las capacidades determinados por la diversidad del tejido social de una comunidad, se han convertido en una acción de innovación cívica a partir de la sinergia de dos elementos centrales: 1) la apropiación de las tecnologías como un medio y un recurso, y 2) el ejercicio y empoderamiento ciudadano a través de procesos online y offline. En la primera de las iniciativas, los Retos Cívicos, podemos identificar que sus características principales son la autonomía, la autogestión, es decir, se trabaja desde y para la ciudadanía. Este modelo faculta y propicia el empoderamiento y la autotransformación, es decir "capacita a los ciudadanos para decidir sobre su destino, inquietudes y oportunidades con la mediación de la tecnología individual para innovar socialmente" (Fernández, 2011).

En la segunda de las iniciativas, los Retos Públicos, el modelo se centra en la gestión y transparencia de un mecanismo de compra de servicios innovadores de tecnología para el gobierno federal orientado al incentivo de las pequeñas y medianas empresas mexicanas del sector. Bajo esta premisa los beneficios y las repercusiones en los procesos de participación ciudadana se reducen a la participación empresas y desarrolladores, por lo que podría cuestionarse si realmente existe un impacto cívico de este modelo. La respuesta es que sí, puesto que la innovación cívica se traduce en innovación publica, la cual hace uso de las tecnologías para "crear o mejorar los canales de comunicación que facilitan la interacción entre los ciudadanos y los gobiernos o el sector privado" (World Bank Group, 2016).

Hemos mostrado dos ejemplos de innovación cívica, innovación que no debe limitarse, ni estancarse, ni tomarse como receta, así como tampoco lo debe hacer la participación y la exigencia ciudadana para la solución y mejora de sus problemáticas y necesidades. Sirvan estas experiencias de Codeando México para motivar el desarrollo de nuevas iniciativas, nuevas comunidades, nuevas redes, nuevos conocimientos y nuevas experiencias, pero sobre todo para la solución de viejos y nuevos retos a las problemáticas sociales y ciudadanas que nos aquejan en cada una de nuestras latitudes.

\section{Agradecimientos}

Mi especial reconocimiento a Codeando México ya todas sus comunidades por el trabajo desinteresado 
que han venido desarrollando a lo largo de estos 4 años de existencia. Agradezco la oportunidad de formar parte de sus comunidades, así como poder apoyar y contribuir en sus proyectos.

Gracias a Miguel Salazar (@miguelsalazarg), Director Ejecutivo de Codeando México, por la invitación, la camaradería y el interés con el que me recibió y me motivó a participar en Codeando México.

\section{Referencias}

Aguilar, A. (2013, marzo 17). Cancelación contrato a Pulso Legislativo por app de 115MDP para Diputados. Recuperado de https://www.change.org/p/c\%C3\%A1mara-de-diputados-m\%C3\%A9xicocancelaci\%C3\%B3n-contrato-a-pulso-legislativo-por-app-de-115mdp-para-diputados

Arellano-Valdivia, J. (2012a, noviembre 23). Desarrollando América Latina 2012. Recuperado de http:// arellanojuan.com/desarrollando-america-latina-2012/

Arellano-Valdivia, J. (2012b, diciembre 30). Desarrollando América Latina - Ganadores regionales. Recuperado de http://arellanojuan.com/desarrollando-america-latina-ganadores-regionales/

Arenilla-Sáez, M. \& García-Vegas, R. (2013). Innovación social. La integración social en la administración pública. Madrid: INNAP-Netbiblio. [Libro Electrónico]

Casanueva, J. M. (2013a, abril 8). \#app115 un hito para abrir el gobierno un bit a la vez. Recuperado de http://socialtic.org/blog/app1 15-un-hito-para-abrir-el-gobierno-un-bit-a-la/

Casanueva, J. M. (2013b, agosto 12). La apertura del gobierno hacia el emprendimiento. Recuperado de http://socialtic.org/blog/la-apertura-del-gobierno-hacia-el-emprendimiento/

Casanueva, J. M. (2014). Lecciones desde y para el hacking cívico. Blog de SocialTIC. Recuperado de http://socialtic.org/blog/lecciones-desde-y-para-el-hacking-civico/

Codeando América Latina. (2012). Codeando LATAM 2012. Recuperado de http://www.screencast. com/t/Rn8kKE50o92

Codeando México. (2013a, marzo 15). Derrocando a la Mexican tech mafia. Recuperado de https:// web.archive.org/web/20131025035449/http://blog.codeandomexico.org/post/45466651453/ derrocando-a-la-mexican-tech-mafia

Codeando México. (2013b). Reto SAT Móvil: Emprendimiento por la Innovación Pública. Recuperado de https://codeandomexico.org/retos/45-reto-sat-movil-emprendimiento-por-la-innovacion-publica

Codeando México. (2014a). Escenario actual. Recuperado de https://web.archive.org/ 
web/20140507021435/http://codeandomexico.org/acerca/

Codeando México. (2014b, agosto 11). Más Retos de Gobierno para La Banda. Recuperado de http:// blog.codeandomexico.org/2014/08/11/retos-de-gobierno-para-la-banda/

Codeando México. (2014c, agosto 13). Ya están listos los Retos de Gobierno para la Banda. Recuperado de http://blog.codeandomexico.org/2014/08/13/listos-los-retos-de-gobierno-para-la-banda/

Codeando México. (2015, noviembre 25). Hacia un nuevo Codeando México. Recuperado de http:// blog.codeandomexico.org/2015/11/25/hacia-un-nuevo-codeando-mexico/

Codeando México. (2016a). Reto Co-Mapp. Recuperado de https://codeandomexico.org/retos/150reto-co-mapp

Codeando México. (2016b, julio 13). Más Tecnología Cívica para la Comunidad Codeando México. Recuperado de http://blog.codeandomexico.org/2016/07/13/mas-tecnologia-civica-para-lacomunidad/

Codeando México. (2016c). Reto Paso Libre. Recuperado de https://codeandomexico.org/retos/151reto-paso-libre

DAL. (2012a). Desarrollando América Latina - 2012. Recuperado de https://web.archive.org/ web/20160521111317/http://2012.desarrollandoamerica.org/

DAL. (2012b). Conoce las apps \#DAL2012. Recuperado de https://web.archive.org/ web/20160520005200 / http://2012.desarrollandoamerica.org/aplicaciones-dal2012/apps-dal2012/

DAL. (2012c). Los campeones regionales de \#DAL2012. Recuperado de https://web.archive.org/ web/20131209030314 / http://2012.desarrollandoamerica.org/desarrollando-america-latina-2012ya-tiene-campeones-regionales/

DAL.(2012d).CodeandoAméricaLatina. Recuperadodehttps://web.archive.org/web/20130223084639 / http://2012.desarrollandoamerica.org:80/portfolio/codeando-mexico-2/

Di Siena, D. (2015). Civic innovation and civic design. Recuperado de http://www.laciudadviva.org/ blogs/?p=28609

Entrepreneur. (2013, julio 12). Semana del Emprendedor 2013. Recuperado de https://www. entrepreneur.com/article/266122

Fernández, I. (2011). ¿Y tú como ves el empoderamiento? En Innovación para el empoderamiento ciudadano a través de las TIC (pp. 129-132). Madrid: Fundación Cibervoluntarios. Recuperado de http:// 
empodera.org//wpcontent/ uploads/2016/07/empodera_2011.pdf

Gobierno de Jalisco. (2016). Retos Jalisco. Recuperado de https://retos.jalisco.gob.mx/

Gobierno de México. (2013a, agosto 06). \#RetoSATmovil. Recuperado de https://www.gob.mx/ mexicodigital/articulos/retosatmovil?idiom=es

Gobierno de México. (2013b, agosto 10). Presentan a finalistas del Reto SAT Móvil. Recuperado de https://www.gob.mx/sat/prensa/com2013_38?idiom=es

Gobierno de México. (2014a). Lanzamiento de Retos Públicos. Recuperado de https://youtu.be/ RFa1 rgBKPXE

Gobierno de México. (2014b). Alianza por el Gobierno Abierto. Plan de Acción 2013-2015|México. Una nueva relación entre sociedad y gobierno. Recuperado de http://www.opengovpartnership.org/sites/ default/files/pa_aga_2015\%20(1).pdf

Gobierno de México. (2014c). Coordinación de Estrategia Digital Nacional. Acerca de Retos Públicos. Recuperado de http://retos.datos.gob.mx/acerca

Gobierno de México. (2016a). Acuerdo por el que se modifican las políticas y disposiciones para la Estrategia Digital Nacional. Recuperado de http://dof.gob.mx/nota_detalle.php?codigo=5424367\&fe cha $=04 / 02 / 2016$

Gobierno de México. (2016b). En el 4. Informe de Gobierno, conoce algunos de los resultados más importantes de la EDN. Recuperado de https://www.gob.mx/mexicodigital/articulos/en-el-4toinforme-de-gobierno-conoce-algunos-de-los-resultados-mas-importantes-de-la-edn?idiom=es

Gobierno de San Pedro Garza García. (2016). Desafío SP. Recuperado de http://desafios.sanpedro. gob.mx/

Gutiérrez, P. \& Ocejo, A. (2015). México: Informe de avance 2013-2015. Mecanismo de Revisión Independiente. Centro de Contraloría Social y de Estudios de la Construcción Democrática (CCS CIESAS). Recuperado de http://www.opengovpartnership.org/sites/default/files/Mexico_2nd\%20 IRM\%20report_final_1.pdf

Jorge, J. E. (2013). Comunidad cívica y capital social. Questión. Revista especializada en periodismo y comunicación, 1(40), 101-111. Recuperado de http://sedici.unlp.edu.ar/bitstream/ handle/10915/32751/Documento_completo.pdf?sequence=1

MEPP. (2014). Mexican open data initiative supported by MEPP is showcased in Central América. Recuperado de https://propem.org/news_events/mexican-open-data-initiative-supported-propem- 
showcased-central-america/

Morato, M. (2014, agosto 22). El reto de los retos públicos en México (P. Flores, Entrevistador) [Archivo electrónico]. Recuperado de https://www.fayerwayer.com/2014/08/el-reto-de-los-retos-publicos-fwinterviu/

Morato, M. (2015). Los hackers cívicos mexicanos. Recuperado de https://www.fayerwayer. com/2015/01/los-hackers-civicos-mexicanos/

Moreno, A. (2012, septiembre 29). Desarrollando América Latina 2012. Recuperado de http:// applicantes.com/desarrollando-america-latina-2012/

Nava, M. (2013, marzo 15). Diputados gastan 115 mdp en servicios que ya tienen. Recuperado de http://www.animalpolitico.com/2013/03/diputados-contratan-servicios-que-ya-tienen-en-lacamara/\#axzz2PHoqbPuV

Odum, E. P. (1969). The Strategy of Ecosystem Development. An understanding of ecological succession provides a basis for resolving manıs conflict with nature. Science, 164(3877), 262-270. doi:10.1126/science.164.3877.262. Recuperado de http://habitat.aq.upm.es/boletin/n26/aeodu. en.html\#fntext-2

Observatorio del Derecho a la Vivienda. (2016). CO-MAPP. Recuperado de http://www. observatoriodevivienda.org/news/co-mapp/

OpenData.MX. (2012). Resultados DAL2012 México. Recuperado de https://web.archive.org/ web/20131228050026/http://opendata.mx/noticias/163/

Paniagua, M. R. (2013, abril 14). Bringing Down The Mexican Tech Mafia: How Hackers Stopped A \$9.3 Million Fraud. Recuperado de https://techcrunch.com/2013/04/14/bringing-down-the-mexicanmafia-how-mexican-hackers-stopped-a-93-million-fraud/

Phills, J. A., Deiglmeier, K. \& Miller, D. T. (2008). Rediscovering Social Innovation. Stanford Social Innovation Review, Fall, 34-43. Recuperado de http://ssir.org/articles/entry/rediscovering_social_ innovation

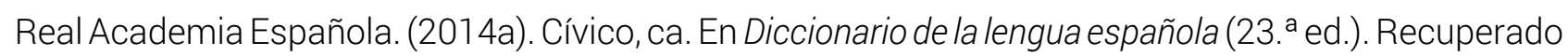
de http://dle.rae.es/?id=9NIY99M

Real Academia Española. (2014b). Civismo. En Diccionario de la lengua española (23. ${ }^{a}$ ed.). Recuperado de http://dle.rae.es/?id=9NzKvPm

Real Academia Española (2014c). Innovación. En Diccionario de la lengua española (23. ${ }^{a}$ ed.). 
Recuperado de http://dle.rae.es/?id=LgxOcfV

Saavedra, Y. (2013, julio 31). El Servicio de Administración Tributaria en México convoca a rediseñar su aplicación móvil. Recuperado de https://hipertextual.com/2013/07/reto-sat-movil

Saúl-Rodríguez, L. \& Morales, P. (2013, abril 5). Presentan 5 aplicaciones gratis para el Congreso. Recuperado de http://www.animalpolitico.com/2013/04/presentan-5-aplicaciones-gratis-para-elcongreso/

Salazar, M. (2015, octubre 15). El 'hacking cívico' como forma de participación ciudadana. Recuperado de http://altavz.com/2015/10/15/el-hacking-civico-como-forma-de-participacion-ciudadana/

Salazar, M. (2016, mayo 25). Codeando México: hackers por la participación ciudadana (D. PérezDamasco, Entrevistador) [Archivo electrónico]. Recuperado de http://distintaslatitudes.net/codeandomexico-hackers-la-participacion-ciudadana

Tecayehuatl, E. (2013, marzo 3). 115 millones por una aplicación o la necesidad de servicios web útiles en México. Recuperado de http://es.gizmodo.com/app115-los-costos-de-una-aplicacion-y-lanecesidad-de-468847399

Torreblanca, E. (2013, agosto 6). Nace la semana del emprendedor. Recuperado de http://www. elfinanciero.com.mx/opinion/nace-la-semana-del-emprendedor.html

Vela, L. (2015). ¿Qué es el hacking cívico y cómo puedes involucrarte? Recuperado de http://www. dineroenimagen.com/2015-06-12/56978

Wilhelmy, R. (2013, abril 19). Codeando México: Hacking Cívico (M. Torres, Entrevistadora) [Archivo electrónico]. Recuperado de https://medium.com/re-magazine/codeando-mexico-hacking-civico47010bc4da7a\#.8di6yyc59

World Bank Group. (2016). Evaluating Digital Citizen Engagement. A practical guide. Washington, D. C.: World Bank. Recuperado de https://openknowledge.worldbank.org/handle/10986/23752 